\title{
Using Linearization and Penalty Approach to Solve Optimal Shape Design Problem with an Obstacle
}

\author{
Alireza Fakharzadeh Jahromi ${ }^{1}$ \\ Dept. of Maths, Faculty of Basic Sciences, Shiraz University of Technology, Shiraz, Iran, P. O. Box, 71555. 313. \\ a_fakharzadeh@sutech.ac.ir \\ Hajar Alimorad, Zahra Rafiei \\ Dept. of Maths, Faculty of Basic Sciences, Shiraz University of Technology, Shiraz, Iran \\ h.alimorad@sutech.ac.ir \\ z.rafiei@sutech.ac.ir
}

Article history:

Received February 2013

Accepted March 2013

Available online April 2013

\begin{abstract}
To obtain the best domain of an elliptic boundary control problems, with and without abstacle, two approches are presented. The based measure method, apply a linearization technique and find the optimal domain and trajectory via a solution of finite linear method by using an optimization search technique. In the second one, by introducing the penalty function and then emplaying the finite element method the optimal domain for the same problem determind. The comparison between two methods is done via presenting some numerical simulations.
\end{abstract}

Keywords: Shape optimization; Measure; Penalty approach; Finite element method; obstacle.

\section{Mathematics Subject Classification: Primary 49J20; Secondary 65 K99}

\section{Introduction and Preliminaries}

Optimal shape design(OSD) theory, generally deals with finding the best domain for a system of differential equations and also perhaps its involved control variables. This kind of problems has applicated in many branch of sciences and engineering like aerospace, automotive industry, structural mechanics and etc. In this manner, many methods have been created specially for solving such problems; the level set method [7], mapping method [4], penalization approach [5], topology method [10] and the controllability approach [3] are some of these kind of methods. Also, based measure method, which we call the shape-measure method, is one of them that presents a linear treatment based on the properties of positive Radon measures; for instance in [2] the shape-measure method was presented for systems governed by elliptic equations in cartesian coordinates. In that paper the process of solution was achieved in two stages. First for a fixed domain, by using the density property and the idea of approximating a curve by broken

\footnotetext{
${ }^{1}$ Corresponding author
} 
lines, the boundary of domain could be determined with fixed number of points (say Mrepresentation). Then unknown domain could be considered as a function of $\mathrm{M}$ variables.

In this work, first, we extended and improved this method to solve the problems in the presence of obstacles. For this purpose, we impose the elliptic OSD problem some new constraint. Secondly, by using the penalty method and considering an initial domain, we employ the finite element approach to divide the domain into a finite number of triangles; next, on each of these triangles, we define two variables polynomials which are uniquely defined by their values at the three vertices of a triangle to replace the unknown domain by a piecewise linear arc. As will be explained in section 4, we specify the optimal domain for two cases of presence and abcence of obstacle. By presenting some examples, we compare the shape measure method with the penalty approach to dettermine the advantages of these two methods.

\section{General optimal shape problem}

Let $D \subset R^{2}$ be a bounded domain with a piecewise-smooth, closed and simple boundary $\partial D$ which consists of a fixed and a variable part. The free boundary part, $\Gamma$ is located between the two given points $A$ and $B$ (see figure1); as mentioned in [6], $\Gamma$ can be approximated by line segments between the unknown corners $A_{i}^{\prime} s$ with the fixed y-components $Y_{i}^{\prime} s$ for $i=1,2, \ldots, M$. Definition 2.1. For the given functions $f \in C(D \times R)$ and $g \in C(D)$, the domain $D$ is called admissible if

$$
\Delta u(X)+f(X, u)=g(X),
$$

with the boundary condition $\left.u\right|_{\partial D}=v$, has a bounded solution on $D$, where are the pair $v: \partial D \rightarrow$ $R$ and $u: D \rightarrow R$ are control and trajectory functions.

Let $f_{1}: D \times U \rightarrow R$ and $f_{2}: \partial D \times V \rightarrow R$, be two known continues functions. Then, in general, we study the following optimal shape design problem over the set of all admissible pairs of domains and controls $(D, u)$ :

$$
\begin{aligned}
& \text { Min: } I(D, v)=\int_{D} f_{1}(X, u(X)) d X+\int_{\partial D} f_{2}(s, v(s)) d s \\
& \text { S.to: } \Delta u(X)+f(X, u)=g(X),\left.u\right|_{\partial D}=v .
\end{aligned}
$$

\subsection{The obstacle problem}

To demonstrated the abilities of shape-measure method under the conditions of existing some obstacles, is another purpose of this paper. We consider an area in domain (presented in figure (2)) such that the solution of the above problem in this area is a fixed amount. One application field of this the problem is a specific speed in the exit area of a nozzle [11]. As a whole, the solution of the obstacle problem, is a function that minimizes the integral energy and other forms of objective functions like weight, press, displacement with extra constraint.

Considering the elliptic system (2), the purpose obstacle problem in this paper is the determination of the optimal domain on set $K=\left\{u(X) \in H^{1}(D),\left.u\right|_{\partial D}=v, u \geq \psi\right\}$, where $H^{1}(D)$ is the sobolev space of order 1 . Consuming the given set $S \subset D$ is an area that $u$, should be the fixed function $\psi$ and in the other points the solution select amounts more than $\psi$ (see figure (2)).

\section{Shape-Measure method}

The idea of using measures to determine the solution of optimal shape design problems was based on the embedding procedure which introduced by Rubio in [8]. The first attempt was sucssed in 1996 where the optimal shape was created in polar coordinates as a mathematical problem under special circumstances in [1]. Then the method was extended to determine the best domains for different systems and the verify of application like [11]. The method, which for simplicity we call shape measure, has many advantages like an automatic existence theorem, 
ability of finding some how the global solution, and linearity. In this section we briefly explain this procedure and the reader can find more details in the other related literature.

By multiplying two sides of (1) by the function $\varphi \in H_{0}^{1}(D)$, and then, integrating over $D$ and using Green formula [13], the general differential equation (1) is converted to the following generalized problem:

$$
\int_{D}(u \Delta \varphi+\varphi f) d X+\int_{\partial D} v(\nabla \varphi \cdot n) d s=\int_{D} \varphi g d X, \quad \forall \varphi \in H_{0}^{1}(D)
$$

Here, $H_{0}^{1}(D)=\left\{\varphi \in H^{1}(D):\left.\varphi\right|_{\partial D}=0\right\}$.

We define two following positive linear functionals:

$$
u(.): F \rightarrow \int_{D} F(X, u(X)) d X ; v(.): G \rightarrow \int_{\partial D} G(s, v(s)) d s .
$$

Then, Riesz Representation Theorem [13] is applied to transfer (3) into the space of positive Radon measure $M^{+}(\Omega) \times M^{+}(\omega)$, where $\Omega=D \times U$ and $\omega=\partial D \times V$ and

$$
\begin{gathered}
\mu(F)=\int_{\Omega} F d \mu=\int_{D} F(X, u(X)) d X, \forall F \in C(\Omega) ; \\
\nu(G)=\int_{\omega} G d v=\int_{\partial D} G(s, v(s)) d s, \forall G \in C(\omega) .
\end{gathered}
$$

We have added two set of constrains to the problem to ensure that the projection of $\mu$ and $\lambda$ on $(x, y)$ plane and real line is a lebegue measure:

$$
\begin{gathered}
\mu(\xi)=\int_{D} \xi(x, y) d x=a_{\xi}, \quad \forall \xi \in \mathrm{C}(\Omega) ; \\
\vartheta(\tau)=\int_{\partial D} \tau(s) d s=b_{s}, \forall \tau \in \mathrm{C}(\omega) .
\end{gathered}
$$

Extending the underlying space, applying density properties in the involved spaces and using linear properties of atomic measures accompany with two stages of approximation, cause to find the nearly optimal solution via the following finite linear programming problem [2]:

Min: $I\left(D, v_{D}^{*}\right)=\sum_{n=1}^{N} \alpha_{n} f_{1}\left(Z_{n}\right)+\sum_{k=1}^{K} \beta_{k} f_{2}\left(Z_{k}\right)$

S.to: $\sum_{n=1}^{N} \alpha_{n} F_{i}\left(Z_{n}\right)+\sum_{k=1}^{K} \beta_{k} G_{i}\left(z_{k}\right)=c_{i}, \quad i=1,2, \ldots, M_{1}$;

$\sum_{n=1}^{N} \alpha_{n} \xi\left(Z_{n}\right)=a_{j}, \quad j=1,2, \ldots, M_{2}$;

$\sum_{k=1}^{K} \beta_{k} \tau\left(z_{k}\right)=b_{k}, \quad k=1,2, \ldots, M_{3}$.

$\alpha_{n} \geq 0, \quad n=1,2, \ldots, N$;

$\beta_{k} \geq 0, \quad k=1,2, \ldots, K$.

Here, $F_{i}=u \Delta \varphi_{i}+\varphi_{i} f, G_{i}=v\left(\nabla \varphi_{i} . n\right), c_{i}=\int_{D} \varphi_{i} g, Z_{n}$ and $z_{k}$ are members of the dense subset of $\Omega$ and $\omega$ respectively, $a_{j}=a_{\xi_{j}}, b_{k}=b_{\tau_{k}}, \alpha_{n}$ and $\beta_{k}$ are unknown coefficient. We remined that by the explained maner in [8], one construct the optimal trajectory and control functions via the obtianed optimal coeficients from (4). Now, the shape measure method is applied in two steps. First for a given $D$ one can calculate the value of $I\left(D, v_{D}^{*}\right)$ by solving (4). In this manner, the suboptimal control function, $v_{D}^{*}$, can be identified as the way as described in [1]. In general, for an unknown domain $D$, we approximate its variable part with $M$ segment lines (as mentioned in section 2, figure 2). Consequently, to identify a representative domian set approximately, one can determine the finite number of corners. Thus the vector function $J: D \rightarrow$ $I\left(D, v_{D}^{*}\right)$ can be identified as a function of $M$ variable in which for any given domain $D, J(D)$ can be calculated by solving (4). Therefore, one can obtain the optimal pair of domain and its related optimal control by optimizing J(D) with a suitable search techniques. The following theorem will show that the obtained domain and control is optimal.

Theorem 3.1. Let the search algorithm give the global minimizer $D^{*}$ for $J$; then the obtained pair of domain and its related optimal control obtained by $(4),\left(D^{*}, v_{D^{*}}^{*}\right)$, is the optimal solution of (1) (see the prove in [2]). 


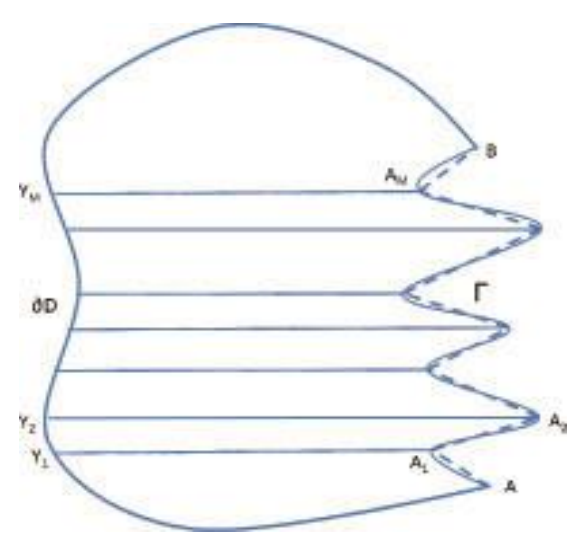

Figure 1: The domain D in general form

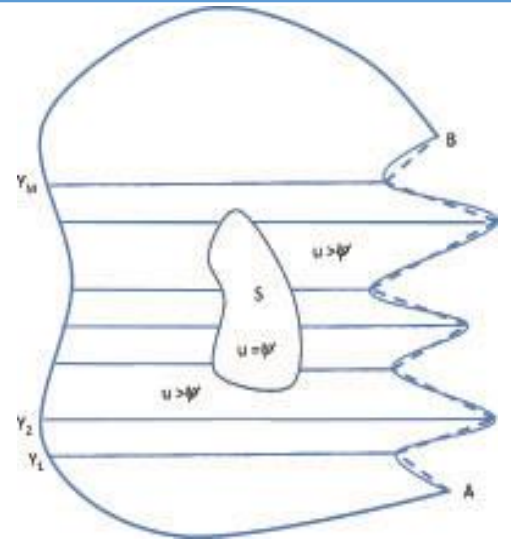

Figure 2: An admissible domain D under the assumptions of the presence of obstacle

\section{Penalty approach method}

In this section, we state the method of solving problem (2) by using penalty function and finite elements method. First, we transfer the problem into a problem with zero boundary condition. Then, to ensure that this problem has a solution, instead of solving it in space $V=H_{0}^{1}(D)$, we demonstrate it on the closed and convex subspace of $V$ which is displayed by $K$. To apply penalty method for solving this problem, first, we must remind some needed definitions for introducing a generalized form of it. For an unknown domain $D$ as shown in figure (1) assume that $v \in$ $H^{1 / 2}(\partial D)$ and $f(x, u)$ is a linear function respect to $u$; since (by the trace theorem Error! Reference source not found.) there exists $\gamma: H^{1}(D) \rightarrow H^{1 / 2}(\partial D)$, (where $H^{1 / 2}(\partial D)$ is the sobolev space in $\partial D$ with fractional derivative) we have a function $G \in H^{1}(D)$ such that $\gamma G=v$. Thus, we can set $u=w+G$ in equation (1), where $w$ solves the problem with homogeneous Dirichlet boundary conditions:

$$
\left\{\begin{array}{l}
\Delta w+f(X, w)=g(X)-\Delta G-f(X, G) \text { in } D \\
w=0 \text { on } \partial D
\end{array}\right.
$$

The corresponding weak formulation of each $p \in H_{0}^{1}(D)$,

$$
\int_{D} \Delta w \cdot p d A+\int_{D} f(X, w) \cdot p d A=\int_{D} g(X) \cdot p d A-\int_{D} \Delta G \cdot p d A-\int_{D} f(X, G) \cdot p d A
$$

by using Green theorem [13] we have:

$$
\int_{D} \Delta w \cdot p d A=\int_{D} \nabla w \cdot \nabla p d A-\int_{\partial D} \frac{\partial w}{\partial n} \cdot p d s=\int_{D} \nabla w \cdot \nabla p d A .
$$

Since $p \in H_{0}^{1}(D)$ thus $p=0$ on $\partial D$, and we have:

$$
\int_{D} \Delta G . p d A=\int_{D} \nabla G . \nabla p d A .
$$

Thus, a weak formulation of problem (5) is obtained as:

$$
\begin{aligned}
& \int_{D} \nabla w \cdot \nabla p d A+\int_{D} f(X, w) \cdot p d A \\
& =\int_{D} g(X) \cdot p d A-\int_{D} \nabla G \cdot \nabla p d A-\int_{D} f(X, G) \cdot p d A .
\end{aligned}
$$

We set:

$$
a(w, p)=\int_{D} \nabla w \cdot \nabla p d A+\int_{D} f(X, w) \cdot p d A,
$$

and

$$
\ell(p)=\int_{D} g(X) \cdot p d A-\int_{D} \nabla G \cdot \nabla p d A-\int_{D} f(X, G) \cdot p d A
$$

Notice that $g(X), G$ and $f(X, G)$ are known.

The finite element method provides a natural framework for finding finite-dimensional approximation of weak solution of elliptic boundary value problems Error! Reference source not found.. Set $V=H_{0}^{1}(D)$ and $a(.,$.$) is:$ 
a) continuous on $V \times V$ (i.e. $\exists M=$ const $>0 \quad|a(u, p)| \leq M\|u\|\|p\| \forall u, p \in V$ ), Since by using Schwarz inequality [13], we have:

$$
\begin{aligned}
& \left|\int_{D} \nabla u \cdot \nabla p d A+\int_{D} f(X, u) \cdot p d A\right| \leq\left|\int_{D} \nabla u \cdot \nabla p d A\right|+\left|\int_{D} f(X, u) \cdot p d A\right| \\
& \leq\left(\left\{\int_{D}|\nabla u|^{2} d A\right\}^{\frac{1}{2}}\right)\left(\left\{\int_{D}|\nabla P|^{2} d A\right\}^{\frac{1}{2}}\right)+\left(\left\{\int_{D}|f(X, u)|^{2} d A\right\}^{\frac{1}{2}}\right)\left(\left\{\int_{D}|p|^{2} d A\right\}^{\frac{1}{2}}\right)
\end{aligned}
$$

where $f(X, u)$ is a bounded function .

b) v-elliptic on $V$ (i.e. if $a(u, u) \geq 0$, then $\exists m>0 \quad \forall p \in V a(p, p) \geq m\|p\|^{2}$ );

if $\int_{D} f(X, p) \cdot p d A$ is positive then :

$$
\int_{D} \nabla p \cdot \nabla p d A+\int_{D} f(X, p) \cdot p d A \geq \int_{D} \nabla p \cdot \nabla p d A=\|p\|^{2} .
$$

and if $\int_{D} f(X, p) \cdot p d A$ is negative then :

$$
\left|\int_{D} \nabla p . \nabla p d A+\int_{D} f(X, p) \cdot p d A\right| \geq\left|-c \int_{D} p d A\right| \geq c\left|\int_{D} p d A\right|=c\|p\|_{1}^{2} .
$$

For a given $\mathrm{N}$-dimensional subspace $V_{N} \subseteq V$, consider the problem

$$
w_{N} \in V_{N}, \quad a\left(w_{N}, p\right)=\ell(p) \quad \forall p \in V_{N}
$$

by considering the above assumptions, Lax-Milgram Lemma guarantees the existence of an unique solution $w_{N}$ for this problem (for more detail see [6]).

Definition 4.1. A triple $(V, a, \ell)$, where $a(.,$.$) is a bilinear form and \ell \in V^{\prime}$ ( $V^{\prime}$ is the dual space of $V$ ), will be called an abstract linear elliptic equation on $V$. An element $w \in V$, which satisfying

will be called a solution of $(V, a, \ell)$.

$$
a(w, p)=\ell(p), \quad \forall p \in V,
$$

Remark 4.2. If $a(.,$.$) is symmetric on V \times V$ i.e. $a(w, p)=a(p, w)$ for any $w, p \in V$, then (9) is equivalent to find $w \in V$ such that $J(w) \leq J(p)$ for any $p \in V$, where $J(p)=(1 / 2) a(p, p)-$ $\ell(p)$ is a quadratic functional (see [9]).

Definition 4.3. A triple $(K, a, \ell)$, where $a(.,$.$) is a bilinear form and \ell \in V^{\prime}$, will be called an abstract linear elliptic inequality on $K$. We also call an element $w \in K$ the solution if the inequality

holds for any $p \in K([9])$.

$$
a(w, p-w) \geq \ell(p-w)
$$

Theorem 4.4. If $a(.,$.$) is a symmetric function on K, \forall w, p \in K$, then (10) is equivalent to the problem of finding a minimizer $w$ of $J$ over $K$ :

(see [9] for proof).

$$
w \in K: \quad J(w) \leq J(p), \quad \forall p \in K
$$

Now we describe the penalty approach; for this purpose, we use penalty function and transfer inequality (10) to an equality one in $V$ that is equal to the problem in $K$. We follow Error! Reference source not found. and present the following definition:

Definition 4.5. Let $j: V \rightarrow \mathbb{R}^{1}$ be a functional satisfying:

(i) $j(v) \geq 0 \quad \forall v \in V$ that $j(v)=0$ iff $v \in K$;

(ii) $j$ is convex, weakly lower semicontinuous on $V$;

(iii) $j \in c^{k}, k \geq 1$, (k times continuously differentiable on $V$ ).

Now, we define problem $\left(P_{\varepsilon}\right)$ by:

$$
\left(P_{\varepsilon}\right)\left\{\begin{array}{l}
\text { Find } u_{\varepsilon} \in \text { Vsuchthat } \\
J_{\varepsilon}\left(u_{\varepsilon}\right) \leq J_{\varepsilon}(v) \quad \forall v \in V,
\end{array}\right.
$$

where $\varepsilon>0$ is a penalty parameter tending to zero and 
$\left(P_{\varepsilon}\right)$ is an optimization problem on $V$ (see[6]).

$$
J_{\varepsilon}(v):=J(v)+\frac{1}{\varepsilon} j(v)
$$

Proposition: If $u_{\varepsilon} \in V$ solves $\left(P_{\varepsilon}\right)$, then

where

$$
J_{\varepsilon}^{\prime}\left(u_{\varepsilon}, v\right)=0 \quad \forall v \in V,
$$

$$
J_{\varepsilon}^{\prime}\left(u_{\varepsilon}, v\right)=\lim _{t \rightarrow 0} \frac{J_{\varepsilon}\left(u_{\varepsilon}+t v\right)-J_{\varepsilon}\left(u_{\varepsilon}\right)}{t}
$$

is the derivative of $J_{\varepsilon}$ at $u_{\varepsilon}$ in the direction of $v$. Taking into account (12), (13) and (14) is equivalent to

$$
a\left(u_{\varepsilon}, v\right)+\frac{1}{\varepsilon} j^{\prime}\left(u_{\varepsilon}, v\right)=\ell(v) \quad \forall v \in V .
$$

Proof: Define $J_{\varepsilon}(v):=J(v)+(1 / \varepsilon) j(v)$ where $J(v)=(1 / 2) a(v, v)-\ell(v)$; thus we have:

$$
\begin{aligned}
& J_{\varepsilon}\left(u_{\varepsilon}+t v\right)=J\left(u_{\varepsilon}+t v\right)+\frac{1}{\varepsilon} j\left(u_{\varepsilon}+t v\right) \\
& =\frac{1}{2} a\left(u_{\varepsilon}+t v, u_{\varepsilon}+t v\right)-\ell\left(u_{\varepsilon}+t v\right)+\frac{1}{\varepsilon} j\left(u_{\varepsilon}+t v\right) \\
& =\frac{1}{2} a\left(u_{\varepsilon}, u_{\varepsilon}\right)+\frac{t}{2} a\left(u_{\varepsilon}, v\right)+\frac{t}{2} a\left(v, u_{\varepsilon}\right)+\frac{t^{2}}{2} a(v, v) \\
& -\ell\left(u_{\varepsilon}\right)-t \ell(v)+\frac{1}{\varepsilon} j\left(u_{\varepsilon}+t v\right) \\
& =\frac{1}{2} a\left(u_{\varepsilon}, u_{\varepsilon}\right)+t \times a\left(u_{\varepsilon}, v\right)+\frac{t^{2}}{2} a(v, v) \\
& -\ell\left(u_{\varepsilon}\right)-t \ell(v)+\frac{1}{\varepsilon} j\left(u_{\varepsilon}+t v\right)
\end{aligned}
$$

then

and

$$
J_{\varepsilon}\left(u_{\varepsilon}\right)=\frac{1}{2} a\left(u_{\varepsilon}, u_{\varepsilon}\right)-\ell\left(u_{\varepsilon}\right)+\frac{1}{\varepsilon} j\left(u_{\varepsilon}\right)
$$

$$
\frac{J_{\varepsilon}\left(u_{\varepsilon}+t v\right)-J_{\varepsilon}\left(u_{\varepsilon}\right)}{t}=a\left(u_{\varepsilon}, v\right)+\frac{t}{2} a(v, v)-\ell(v)+\frac{1}{\varepsilon} \frac{j\left(u_{\varepsilon}+t v\right)-j\left(u_{\varepsilon}\right)}{t} .
$$

If we give limit from both side of equation (16) as $t \rightarrow 0$, we have :

$$
0=J^{\prime}\left(u_{\varepsilon}, v\right)=a\left(u_{\varepsilon}, v\right)-\ell(v)+\frac{1}{\varepsilon} j^{\prime}\left(u_{\varepsilon}, v\right)
$$

thus

$$
a\left(u_{\varepsilon}, v\right)+\frac{1}{\varepsilon} j^{\prime}\left(u_{\varepsilon}, v\right)=\ell(v)
$$

The relationship between $u_{\varepsilon}$ and the solution $w$ of (10) is given by following theorem:

Theorem 4.6. It holds $u_{\varepsilon} \rightarrow w$ in $V$ as $\varepsilon \rightarrow 0$ (see[6]).

If we define the penalty functional " $j$ " by $j(y)=(1 / 2) \int_{\Gamma}\left(y^{-}\right)^{2} d s$, where $y^{-}=(1 / 2)(|y|-y)$, then condition in definition (5.3) are satisfied (see[6]) and

$$
j(y, p)=-\int_{\Gamma} y^{-} p d s .
$$

We solve problem (9) on $K$, since $K$ is closed and convex subset of $V$ and we enure that the problem has one solution in this set, i.e. find $u \in K$ such that :

$$
a(u, y-u) \geq \ell(y-u) ; \quad \forall y \in K \text {, }
$$


where

$a(u, y-u)=\int_{D} \nabla u \cdot \nabla(y-u) d A+\int_{D} f(X, u) \cdot(y-u) d A$

$\ell(y-u)=\int_{D} g(x) \cdot(y-u) d A-\int_{D} \nabla G \cdot \nabla(y-u) d A-\int_{D} f(x, G) \cdot(y-u) d A$.

By using the penalty functional (18) the penalized form of problem (9) can be written as finding $u_{\varepsilon} \in V$ such that :

where

$$
J_{\varepsilon}\left(u_{\varepsilon}\right) \leq J_{\varepsilon}(y) \quad \forall y \in V,
$$

$$
J_{\varepsilon}(y)=J(y)+(1 / 2 \varepsilon) \int_{\Gamma}\left(y^{-}\right)^{2} d s
$$

and

$$
\begin{aligned}
& J(y)=(1 / 2) \int_{D} \nabla u \cdot \nabla y d A+\int_{D} f(X, u) \cdot y d A-\int_{D} g(x) \cdot y d A \\
& -\int_{D} \nabla G \cdot \nabla y d A-\int_{D} f(x, G) \cdot y d A .
\end{aligned}
$$

We solve problem (21) by applying approximation finite element method and penalty function (more detail of finite element method in [6]) and definition:

$$
D(\alpha)=\left\{\left(x_{1}, x_{2}\right) \in \mathbb{R}^{2} \mid 0<x_{1}<\alpha\left(x_{2}\right), 0<x_{2}<1\right\},
$$

where $\alpha$ is a positive lipschitz function defined in $[0,1]$ and

$$
\Gamma(\alpha)=\left\{\left(x_{1}, x_{2}\right) \in \mathbb{R}^{2} \mid x_{1}=\alpha\left(x_{2}\right), x_{2} \in(0,1)\right\}
$$

is the unknown boundary. Then we solve problem (21) by the objective function $I(D, u)$ mentioned in (2). Thus, problem (21) is equivalent to

where

$$
\left\{\begin{array}{l}
\text { Find } u_{\varepsilon}(\alpha) \in V(\alpha) \quad \text { suchthat } \\
a\left(u_{\varepsilon}(\alpha), p\right)+\frac{1}{\varepsilon}\left(\rho\left(u_{\varepsilon}(\alpha), p\right)_{\alpha}=\ell(p) ; \quad \forall p \in V(\alpha),\right.
\end{array}\right.
$$

$$
(\rho(y), p)_{\alpha}=-\int_{0}^{1} y^{-}(\alpha) p(\alpha) d x_{2}
$$

Now, we can minimize $I$ with respect to constraint (23) by finite element method. For applying this method, we state unknown function $u_{\varepsilon}(\alpha)$ as follow:

$$
u_{\varepsilon}(\alpha)=\sum_{j=1}^{n} x_{j} \phi_{j}(x)
$$

where $n$ is the amount of the node in triangulation and $\phi_{j}\left(x_{1}, x_{2}\right)=a x_{1}+b x_{2}+c$ is the courant basis function. Since relation (23) satisfied for all $p \in V(\alpha)=H_{0}^{1}(\Omega(\alpha)$ ), we set $p=$ $u_{\varepsilon}(\alpha)$ and we transfer the problem into homogeneous boundary condition, thus, $u_{\varepsilon}(\alpha) \in$ $H_{0}^{1}(\Omega(\alpha))$. We state problem (23) as follow:

$\int_{D} \nabla u_{\varepsilon} \cdot \nabla u_{\varepsilon} d A+\int_{D} f\left(X, u_{\varepsilon}\right) \cdot u_{\varepsilon} d A+\frac{1}{\varepsilon}\left(\rho\left(u_{\varepsilon}, u_{\varepsilon}\right)\right)_{\alpha}$

$=\int_{D} g(X) \cdot u_{\varepsilon} d A-\int_{D} \nabla G \cdot \nabla u_{\varepsilon} d A-\int_{D} f(X, G) \cdot u_{\varepsilon} d A$

$\Rightarrow \sum_{i=1}^{n} x_{i} \sum_{j=1}^{n} x_{j} \int_{D} \nabla \phi_{i} \cdot \nabla \phi_{j} d A+\sum_{i=1}^{n} x_{i} \sum_{j=1}^{n} x_{j} \int_{D} f\left(X, \phi_{i}\right) \cdot \phi_{j} d A+\frac{1}{\varepsilon} D(x(\alpha))$

$=\sum_{i=1}^{n} x_{i} \int_{D} g(X) \cdot \phi_{i} d A-\sum_{i=1}^{n} x_{i} \int_{D} \nabla G \cdot \nabla \phi_{i} d A-\sum_{i=1}^{n} x_{i} \int_{D} f(X, G) \cdot \phi_{i} d A$

where

$$
D(x(\alpha))=-\frac{h}{\varepsilon}\left(0,0, \ldots, 0,\left(x_{n-D(h)+1}^{-}\right)^{2}, \ldots,\left(x_{n}^{-}\right)^{2}\right),
$$

$h$ is the distance between two $x_{2}$ coordinate of consecutive nodes that assume is equal and $D(h)$ is the amount of nodes that have lied on $\Gamma(\alpha)$. we set :

$$
\begin{aligned}
& A(\alpha)=\int_{D} \nabla \phi_{i} \cdot \nabla \phi_{j} d A+\int_{D} f\left(X, \phi_{i}\right) \cdot \phi_{j} d A ; \\
& F(\alpha)=\int_{D} g(X) \cdot \phi_{i} d A-\int_{D} \nabla G \cdot \nabla \phi_{i} d A-\int_{D} f(X, G) \cdot \phi_{i} d A
\end{aligned}
$$

Thus we solve problem in matrix form as follow: 


$$
\left\{\begin{array}{l}
\text { Find } x(\alpha) \in R^{n} \text { suchthat } \\
A(\alpha) x(\alpha)+\frac{1}{\varepsilon} D(x(\alpha))=F(\alpha),
\end{array}\right.
$$

where $x(\alpha)$ are unknowns. In this paper, we use penalty function method and finite element method to solve obstacle optimal shape problems and we present numerical example that are solved by this method and compare conclusion by the shape measure method.

\section{Numerical results}

For the numerical simulations, The fixed part of the domain $D \subset R^{2}$ is supposed to be a union of three segments: part of the line $y=0$ between the points $(0,0)$ and $A=(1,0)$; part of the line $x=0$ between the points $(0,0)$ and $(0,1)$, and part of the line $y=1$ between the points $(0,1)$ and $B=(1,1)$. The variable (free boundary) part is a curve $\Gamma$ with the initial and final points $A$ and $B$ respectively, so that $\partial D$ is a simple and closed curve (see figure (1) and (2)). In the following we present three example on this domain to obtain the optimal unknown boundary and its related control for the described elliptic system in (2). In the first, one, no obstacle is existed on domain and the second constrains an obstacle. Both are solved by the two described methods in sections 3 and 4 and the obtained optimal results are compare.

Example 5.1. To use the shape measure method, we assumed $\mathrm{M}=5, \mathrm{Y} 1=0.2, \mathrm{Y} 2=0.35, \mathrm{Y} 3=0.5$, $\mathrm{Y} 4=0.65, \mathrm{Y} 5=0.8$ and $f_{1}(X, u)=(u-0.1)^{2}$ and $f_{2}(s, v)=0$. Then, as explained in section 3 , by selecting $M_{1}=3, M_{2}=5, M_{3}=5$ the related approximated relax problem like (4) is constrained with is constraint and $\alpha_{n}$ are variables. We select $\mathrm{N}=550$ nodes $Z_{n}=\left(x_{n}, y_{n}, u_{n}\right)$ in $\Omega$ and $\mathrm{K}=110$ nodes $Z_{k}=\left(s_{k}, v_{k}\right)$ in $\omega$.For this example we chose. The variables are supposed to satisfy $0 \leq$ $X_{m} \leq 2, m=1,2, \ldots, 5$. We used Nelder-Mide algorithm for find the optimal domain. In applying shape measure method, we solve linear programming problem and performance time is 15 minute. The optimal value of cost function was obtained 0.002810581141213 . The optimal domain is presented in figure (3).

By solving the above problem with penalty method and using finite element method, we solve nonlinear programming problem (24) and obtain the optimal vector $X$; then, the unknown boundary $\Gamma(\alpha)$ is obtained by the optimal component of vector $X$ (coefficient of courant basis function) that lie on $\Gamma(\alpha)$ ). The optimal value of objective function is $I=0.03554$ and optimal shape is presented in figure (4). In this method performance time is 30 minute. The value of objective function in shape measure method is less than that one in penalty approach.

In following examples we solve optimal shape design for obstacle problem with shape measure method and finite element method.

Example 5.2. In this example in addition to constraint (2), we have constrains $\left.u\right|_{\partial D}=v$ and $u \geq$ 0 too. In domain $S$ which here is a circle with the center $(1 / 2,1 / 2)$ and radius $1 / 4$, function $u$ is equal to zero and in domain $D-S, u \geq 0$. Region $S$ is completely in $D$; therefore, we add condition $0.7 \leq x_{m} \leq 2$ to the constrains of the problem and for the points which are in $S, u=$ 0 and for those which are in $D-S, u \in(0,1)$. For the penalty method, we also add the constraints $0.7 \leq x_{m} \leq 2$. In order to satisfy condition $u=0$ in domain $S$, for the nodes which are in this domain, we set $x_{j}=0$. For the rest nodes, we set $\sum_{j=1}^{n} x_{j} \varphi_{j} \geq 0$. Additionally, since domain $D$ is in the first quarter of plane, therefore, $x_{1} \geq 0$ and $x_{2} \geq 0$. We use this quality to specify the sign of $\varphi_{j}$.

The obtained numerical results by using shape measure method is :

the optimal value of $I(D, v)=0.565997500$;

the value of the variables in the final step:

$$
X_{1}=0.0624, X_{2}=0.7375, X_{3}=0.8091, X_{4}=0.7885, X_{5}=0.0275 \text {. }
$$


the optimal domain is presented in figure(5). We solve this problem by finite element method, the optimal value of objective function is $I=0.45698$ and the obtianed optimal shape is presented in figure(6).

In this example, the performance time of the program is less than that of penalty method; however, in the latter method, the value of objective function is less than one in the former method.

Example 5.3. In this example, we want that the square $C=[1 / 4,3 / 4] \times[1 / 4,3 / 4]$ be in the optimal domain; so, we add constraint $0.7 \leq x_{m} \leq 2$ to the problem. Besides, we set $g(x, y)=$ 1 , where the points are in $D \cap C$; otherwise, $g(x, y)=0$. We consume that the function $g(X)$ (the fixed control function) is defined as:

$$
g(x, y)= \begin{cases}1 & \text { if }(x, y) \in D \cap C \\ 0 & \text { otherwise }\end{cases}
$$

Where $C$ is the square $\left[\frac{1}{4}, \frac{3}{4}\right] \times\left[\frac{1}{4}, \frac{3}{4}\right]$ and $\left.u\right|_{\partial D}=0, f_{1}=(u-0.1)^{2}$ and $f_{2}=0$. We use the initial values $X_{m}=1.1, m=1,2, \ldots, 8$, as a given domain for starting algorithm. We solved this problem by shape measure method and obtain $I=0.85045$. The optimal shape is presented in figure(7). We solved this problem by using finite element method and obtain $I=0.4141$, the optimal shape is presented in figure(8). Average performance time by finite element method is 150 minute and the number of operation is 5000 . In shape measure method, average performance time is 120 minute and the number of operation is 4378 .

\section{6.conclusion}

In this paper, shape measure method and penalty method were studyed used for determining the optimal shape design with and without obstacle problem.

In penalty method, the preciseness of the method depends on the triangulation and the number of points. The more number of these points, the closer the obtained solution will be to the exact solution of the problem. In addition, solving the nonlinear programming is dependent on the initial value of the variable and we may obtain the local minimum using specific initial value; moreover, number request of operations and performance time are high. In shape measure method, the number of constraint and the dimension of the problem are finite and the more number of these constrains, the more exact will be the method. Also, we obtain the approximation solution by solving a linear programming problem which is simply solvable. performance time and number of operations are less than finite element method and obtained solutions are very close to solution obtained by finite element method.

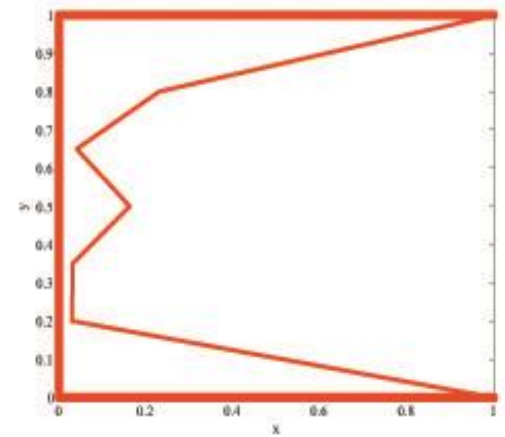

Figure 3: Optimal domain by shape measuremethod for example 5.1

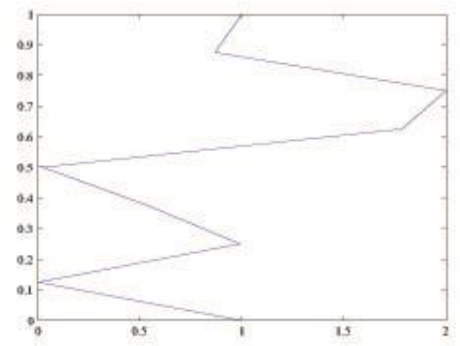

Figure 4:Optimal domain by finite element Method In example 5.1 


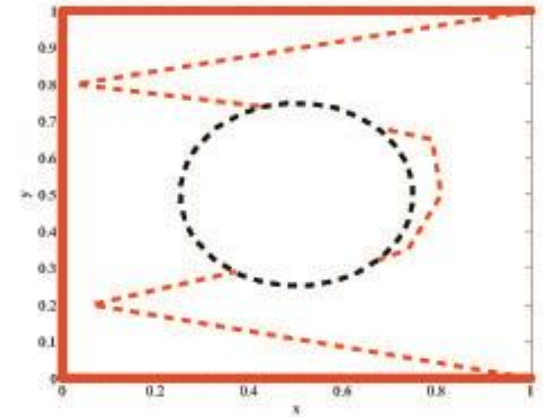

Figure 5: Optimal domain by shape measure Approach for example 5.2
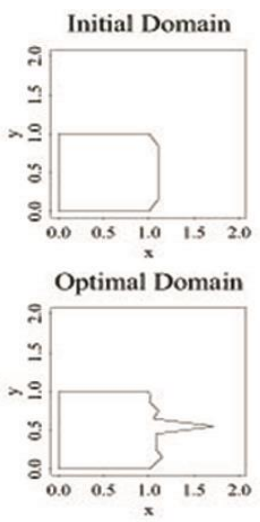

Figure 7: Optimal domain with shape measure method in example 5.3

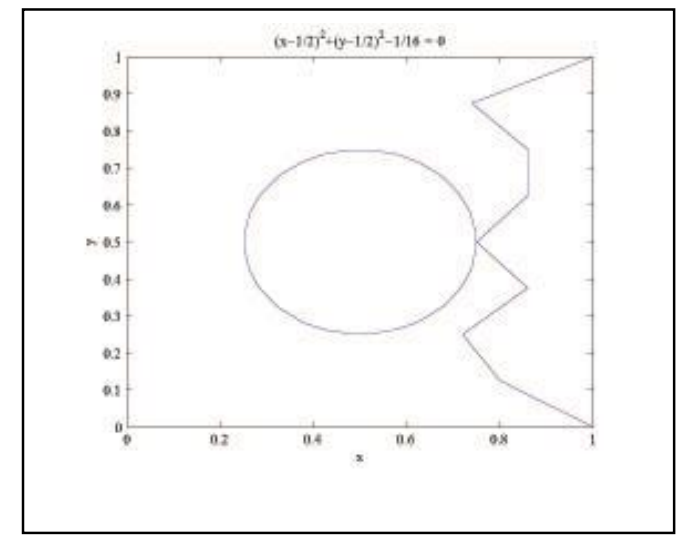

Figure 6: Optimal domain by finite element method in example 5.2

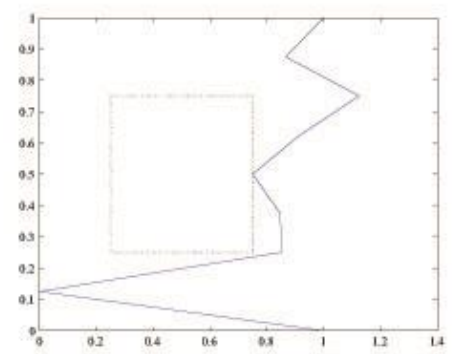

Figure 8:Optimal domain with finite element method for example 5.3

\section{References}

[1] A. Fakharzadeh and J. E. Rubio, Global Solution of Optimal Shape Design Problems, Journal of Analysis and its Application, 18, 143-155 (1999).

[2] A. Fakharzadeh and J.E. Rubio, Best Domain for an Elliptic Problem in Cartesian Coordinates by Means of Shape-measure,AJOP J. of Control, 11, 536-554 ( 2009).

[3] D. Tiba and P. neittanamaki, An embedding of domains in free boundary approach problems and Optimal design,SIAM J. Control optimize. 33, 1587-1602 (1995).

[4] F. Muart and J. Simon, Studies in optimal shape design, Lecture Notes in comput. sci .41, Springer, Berlin, (1976).

[5] H. Kawarada, Numerical solution of a free boundary problem for an ideal fluid, Lecture Notes in phys. 81, Springer, Berlin, (1977).

[6] J. Haslinger and P. Neittaanmaki, Finite Element Approximation for Optimal Shape Design: Theory and Applications, John Wiley and SonSsltd, (1988).

[7] J. A. Sethian, Level set Methods and Fast Marching Methods, University of california, Berkeley, (1999).

[8] J. E. Rubio, Control and Optimization: the linear treatment of nonlinear problems, Manchester university Press, Manchester, (1986).

[9] L. Debnath, and P. Mikusinski, Hilbret spaces with Applications, Elsevier academic Press, 3 rd Edition (2005). 
[10] M. Bendsqe, Optimization of structural topology, shape and material, Springer, New York, (1995).

[11] M. H. Farahi, A. H. Borzabadi, H. H. Mehne and A. V. Kamyad, Measure Theoretical Approach for Optimal Shape Design of a Nozzle,J. Appl. Math and computing, 17, 315-328 (2005).

[12] R. L. Burden and J.D. Faires, Numerical Analysis, Copyright Material, Ninth Edition, (2010).

[13] W. Rudin, Real and Complex Analysis , 2 Edition, Tata McGraw-Hill Publishing Co Ltd., new Dehli, (1983). 